\title{
Reconfigurable antennas and configuration selection methods for MIMO ad hoc networks
}

\author{
John Kountouriotis ${ }^{*}$, Daniele Piazza ${ }^{1,2}$, Prathaban Mookiah', Michele D'Amico² and Kapil R Dandekar ${ }^{1}$
}

\begin{abstract}
In this paper, we quantify the performance enhancements that can be provided by reconfigurable antennas in an ad hoc network where all nodes employ multiple input multiple output communication techniques. We present two types of reconfigurable antennas: a reconfigurable printed dipole array (RPDA), and a reconfigurable circular patch antenna (RCPA). The RPDA and RCPA have a different number of configurations as well as different degrees of pattern diversity between possible configurations. To effectively use these antennas in a network, we introduce and quantify the performance of centralized and decentralized antenna configuration selection schemes for reconfiguration at one or both link ends. We use the sum capacity of the network as a metric to quantify the performance of these antennas in measured and simulated network channels.
\end{abstract}

\section{Introduction}

Research in the area of ad hoc networks has yielded important advances, notably in the field of physical layer techniques. In particular, a lot of effort has been spent in (i) applying smart antennas and antenna diversity techniques to ad hoc networks (e.g., [1-3]), (ii) developing medium access control protocols suitable for multiple input multiple output (MIMO) ad hoc networks (e. g., [4-6]), and (iii) adaptive algorithms for antenna beamforming in ad hoc networks (e.g., [7-9]). Directional antennas, like phased arrays[10,11] and switchable parasitic elements antennas $[12,13]$ have been proposed as a solution to reduce the interference of adjacent nodes, maximizing overall network throughput $[1,3,14]$. In order to further increase the network spectral efficiency, MIMO spatial multiplexing (SM) techniques and diversity techniques have been adopted $[4,5,15]$. However, directional arrays and MIMO SM/diversity techniques cannot be integrated on compact portable devices, where the limited space available renders mounting multiple directional antennas difficult.

In order to overcome practical space limitations and merge the benefits of MIMO SM/diversity techniques with those of directional antennas, we propose to adopt electrically reconfigurable antennas as a key element of MIMO transceivers in ad hoc networks. These antennas

\footnotetext{
* Correspondence: jk368@drexel.edu

'Department of Electrical and Computer Engineering, Drexel University, USA Full list of author information is available at the end of the article
}

have been demonstrated to increase channel capacity while reducing the space occupation of the antenna on the communication device [16-22]. Further, the study that aims to efficiently choose the configuration to be used has started emerging in the literature, like in $[23,24]$. While these previous studies have focused on the performance of reconfigurable antennas in single link communications, there has been no published study on implementing and field testing a system that employs reconfigurable antennas in multi-link MIMO ad hoc networks.

Through this article, we aim to quantify the benefits achievable with reconfigurable antennas in MIMO ad hoc networks, while also investigating antenna configuration selection schemes at each node. In a network scenario, the antenna configuration selection algorithm for a single link not only seeks the configuration combination of both the configuration at the receiver and the configuration at the transmitter, which will provide a "richness", in terms of spatial diversity, channel between the receiver and the transmitter, but will also aim to mitigate the interference that the link is suffering from. This configuration selection process is made more complex by the fact that when the antenna configuration at a transmitter is modified, it changes the interference seen by the other links in the network. While directional antennas can perform interference mitigation by estimating the direction of the incoming signals at the receiver, we show that reconfigurable MIMO antennas 
can achieve a similar result, with lower system complexity, by only estimating the channel matrix.

In order to maximize network sum capacity without a centralized controller, we propose a distributed selection algorithm that can be used to efficiently select the antenna configuration at each node. The performance of this distributed selection scheme is compared to that of an ideal centralized approach that uses an exhaustive search process to assign the optimal antenna configuration to every node. In this article, we assume, for both the centralized and distributed antenna configuration controls, that all transmitters make use of the equal power allocation scheme proposed in [25], which requires no channel feedback from the receiver to the transmitter.

We determine the sum network capacity that can be achieved with MIMO reconfigurable antennas for different network topologies through channel measurements and electromagnetic ray-tracing simulations conducted in an indoor environment. We consider two prototype electrically reconfigurable antenna architectures in a $2 \times$ 2 MIMO system employing SM: (i) a reconfigurable printed dipole array (RPDA) that makes use of two reconfigurable length dipole antennas, and (ii) a reconfigurable circular patch antenna (RCPA) that makes use of a single variable-radius circular patch antenna with two feedpoints. We show that parameters, like the number of antenna configurations, the spatial orthogonality between the array elements and the level of antenna radiation efficiency, can be used to predict the achievable performance with a particular reconfigurable antenna in an ad hoc network.

This article is organized as follows. In Section 2, we introduce the RPDA and RCPA used as a building block of a $2 \times 2$ MIMO ad hoc network and compare and contrast the reconfigurable antenna architectures. In Section 3, the system model adopted to evaluate the performance of the reconfigurable antennas in MIMO ad hoc networks is presented. In Section 4, we propose the distributed and centralized antenna configuration selection scheme for MIMO ad hoc networks employing both reconfigurable antennas and the equal power allocation scheme. In Section 5, we describe the network used for both channel simulations and measurements. Section 6 quantifies the results of the centralized and distributed configuration selection algorithms in the measured and simulated $2 \times 2$ MIMO ad hoc network. Finally, conclusions are drawn in Section 7.

\section{Reconfigurable antenna architectures}

In this section, we present two different compact pattern reconfigurable antennas, intended to be used as a building block of MIMO systems in ad hoc networks. The following antennas were all designed to operate in the 2.4-2.5 GHz frequency band typical of an 802.11-like MIMO network. We quantify the performance of each reconfigurable array using radiation patterns, radiation pattern spatial correlation, and radiation efficiency.

The level of diversity between the patterns generated at the two ports of the array, as well as between the patterns generated at the same port for different configurations of the array, is estimated through the spatial correlation coefficient value [26,27]. Assuming a rich scattering environment, the spatial correlation coefficient, $\rho_{j, k, L, m}$, is defined as [27]

$$
\rho_{j, k, l, m}=\frac{\int_{4 \pi} E_{j, k}(\Omega) E_{l, m}^{*}(\Omega) d \Omega}{\left[\int_{4 \pi}\left|E_{j, k}(\Omega)\right|^{2} d \Omega \int_{4 \pi}\left|E_{l, m}(\Omega)\right|^{2} d \Omega\right]^{1 / 2}}
$$

where $j$ and $l$ define the array ports and $k$ and $m$ the antenna configurations at the port $j$ and $l$, respectively. $E_{j, k}(\Omega)$ is the radiation pattern of the configuration $k$ at port $j$ over the solid angle $\Omega=(\varphi, \theta)$, and $\left\langle^{*}\right\rangle$ is the transpose operator.

Radiation pattern spatial correlation coefficients can be used as a first estimate of the performance of the reconfigurable antenna designs. In particular, the spatial correlation between radiation patterns excited at two different ports of the antenna array gives an indication of the level of decorrelation between the signals collected at the two array elements. A lower correlation coefficient between the two ports will lead to lower correlation between the communication channels from these ports. Similarly, spatial correlation coefficients for radiation patterns generated at the same port for different configurations give an indication of the increment in system diversity achievable using reconfigurable antennas with respect to standard non-reconfigurable antenna systems. The higher the diversity between different configurations, the higher the overall system diversity and the higher the probability of achieving greater channel capacity.

Radiation efficiency is also an important performance measure for reconfigurable antennas. In particular, for a fixed transmitter power, the higher the radiation efficiency, the greater the received signal power and channel capacity. While MIMO channel normalizations sometimes completely remove the effects of antenna radiation efficiency when quantifying the "richness" of the multipath channel, this article does consider the relative efficiencies of the reconfigurable antennas when they are placed in different states.

\section{A. Reconfigurable printed dipole array}

The RPDA, first introduced by the authors in [28], consists of two microstrip dipoles separated by a distance of a quarter wavelength. The active elements in the array can be electrically reconfigured in length using PIN 
diode switches. We define two configurations for each dipole: one in which both switches are activated ("long" configuration), and another in which they are deactivated ("short" configuration). Thus, four different configurations can be defined for the RPDA 2 element array: both antennas "long" (1-l), both antennas "short" (s-s), one antenna "short" and the other "long" (s-l) and vice versa (l-s). A schematic of the structure of the RPDA is depicted in Figure 1a, at the same orientation for the measured patterns. In this schematic, the biasing circuitry is also shown.

The setting of the switches results in different geometries of the antenna and, consequently, in different levels of inter-element mutual coupling and far-field radiation patterns. Four different pairs of radiation patterns can then be produced. Figure $1 \mathrm{~b}$ shows these radiation patterns in the azimuthal plane. It is different from the radiation patterns that appear in [16], where simulated radiation patterns appear, as these patterns are measured patterns in an anechoic chamber of build RPDAs equipped with pin diodes.

Table 1 shows the values of spatial correlation between the measured azimuthal patterns generated at the two ports of the RPDA, while Table 2 shows the values of correlation between the measured azimuthal patterns generated at the same port for all the array configurations. Table 1 shows that the correlation values between radiation patterns at the two ports of the array are small enough for all the configurations $(\leq 0.7)$ to provide significant diversity gain [26]. In contrast to the correlation of the radiation patterns created at the
Table 1 Spatial correlation between patterns generated at two different ports of the RPDA

\begin{tabular}{cccc}
\hline Short-short & Long-short & Short-long & Long-long \\
\hline 0.43 & 0.28 & 0.28 & 0.31 \\
\hline
\end{tabular}

different ports, Table 2 shows that the level of diversity between the different configurations generated at the same port is not high $\left(\rho_{1, k, 1, m}>0.8,[26]\right)$ and is much less than that of the RCPA discussed below.

The radiation efficiency for each array configuration is calculated from the 3D measured radiation patterns according to [29] and is shown in Table 3. It should be noted from this table that there is an imbalance in the radiation efficiency for the different configurations: "short-short" is the most efficient antenna configuration while "long-long" is the least efficient. Differences in radiation efficiency between the array configurations are caused by PIN diode insertion losses. Detailed information about the properties of the RPDA can be found in [16].

\section{B. Reconfigurable circular patch antenna}

The RCPA, introduced by the authors in [18], consists of a circular patch radius of which can be electrically varied by turning all the switches on and off simultaneously. Thus, the RCPA has two configurations: one in which all the switches are turned off, and the electromagnetic mode $T M_{31}$ is excited ("Mode 3" configuration), and another in which they are turned on and the electromagnetic mode $T M_{41}$ is excited ("Mode 4"

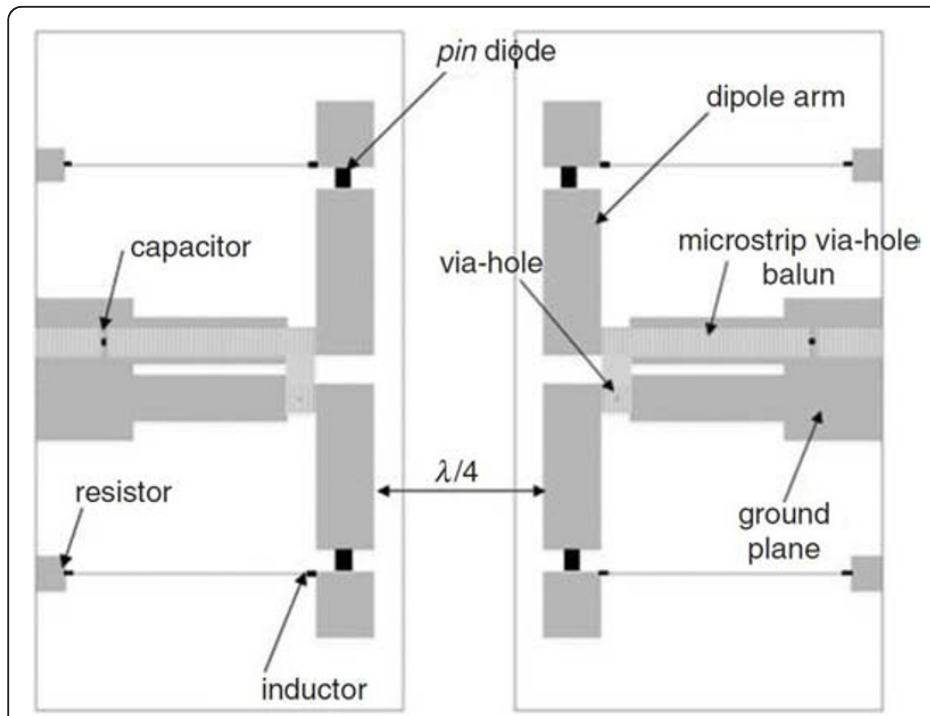

(a)

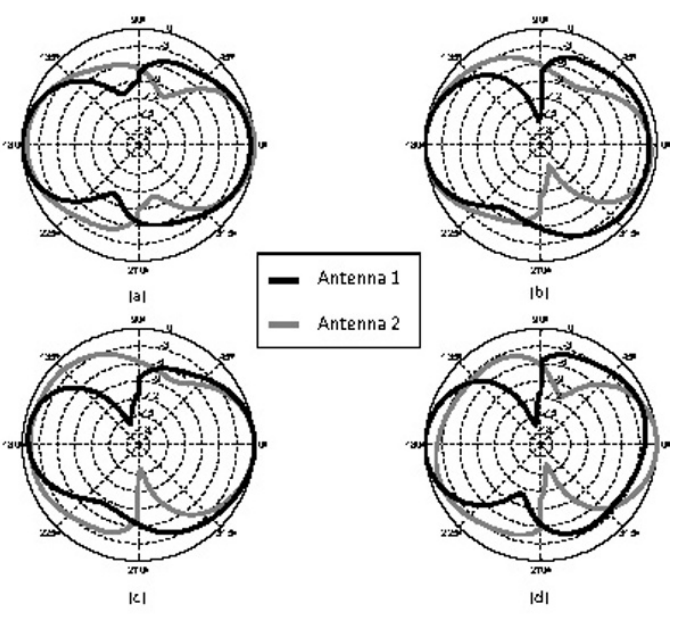

(b)

Figure 1 (a) Schematic of the reconfigurable printed dipole array and (b) radiation pattern (in $\mathrm{dB}$ ) in the azimuthal plane of the two printed dipoles separated by $\lambda / 4$ in all the configurations for an operation frequency of $2.48 \mathbf{~ G H z : ~ ( a ) ~ a n t e n n a ~ 1 , ~ " s h o r t , " ~ a n t e n n a ~ 2 , ~}$ "short"; (b) antenna 1, "long," antenna 2, "short"; (c) antenna 1, "short," antenna 2,"long"; (d) antenna 1, "long," antenna 2, "long". 
Table 2 Spatial correlation between patterns generated at the same port of the RPDA

\begin{tabular}{|c|c|c|c|c|}
\hline & $E_{1, s-s}$ & $E_{1, s-1}$ & $E_{1,1-s}$ & $E_{1, I-I}$ \\
\hline$E_{1,5-s}$ & 1 & 0.87 & 0.94 & 0.9 \\
\hline$E_{1,5-1}$ & 0.87 & 1 & 0.9 & 0.93 \\
\hline$E_{1, \mathrm{l}-\mathrm{s}}$ & 0.94 & 0.9 & 1 & 0.93 \\
\hline$E_{1, s-1}$ & 0.9 & 0.93 & 0.93 & 1 \\
\hline
\end{tabular}

configuration). The structure of the RCPA is shown in Figure 2a. The antenna is fed through two ports placed on the antenna structure such that (i) the radiation patterns excited simultaneously at the two ports are spatially orthogonal to each other and (ii) the port isolation is higher than $20 \mathrm{~dB}$. The design is ideal for compact MIMO systems in that two channels can be achieved using a single physical antenna.

The measured radiation patterns of the RCPA, equipped with pin diodes, are shown in Figure $2 \mathrm{~b}$ for both configurations in the azimuthal plane. These radiation patterns are different from the ones appear in [18], where simulated patterns appear. The figure shows that the radiation patterns excited by "Mode 3 " and "Mode 4" configurations are significantly different, resulting in a large amount of pattern diversity.

The spatial correlation coefficient value between azimuthal patterns generated at two antenna ports and between azimuthal patterns of different configurations generated at the same port, are calculated according to (1) and reported in Tables 4 and 5, respectively. From Table 4, we see that the patterns generated at the two ports of the RCPA are spatially orthogonal for both configurations. Moreover, Table 5 shows the very high level of diversity $\left(\rho_{1, k, 1, m}=0.2\right)$ existing between the two configurations of the RCPA.

The measured antenna radiation efficiencies are reported in Table 6 . This antenna suffers from low radiation efficiency because higher-order modes are excited on a lossy substrate (FR4 with $\tan \delta=0.02$ ). "Mode 3" exhibits higher radiation efficiency than "Mode 4" configuration because lower-order modes are more efficient than higher-order modes [30,31], and because no power is lost in the switches when "Mode 3" is active. For more information on the properties of the RCPA antenna, please refer to [18].

Table 3 Measured radiation efficiency of the RPDA of the RPDA

\begin{tabular}{ccc}
\hline & Antenna 1 (\%) & Antenna 2 (\%) \\
\hline Short-short & 84 & 75 \\
\hline Short-long & 77 & 52 \\
\hline Long-short & 48 & 77 \\
\hline Long-long & 52 & 51 \\
\hline
\end{tabular}

\section{Comparison of RCPA with RPDA}

A comparison between the RPDA and the RCPA shows that, based on the results of Tables 1 and 2 and 4 and 5 , in a rich-scattering environment, the RCPA provides a higher degree of diversity for all its configurations (and among the different configurations) with respect to the RPDA. Therefore, the RCPA allows for higher decorrelation between signals at the receiver and provides higher system diversity. In contrast, the RPDA allows for switching between double the number of radiation patterns offered by the RCPA. Thus, the RPDA and RCPA can be viewed as representing two different "philosophies" for using reconfigurable antennas in wireless communications systems: (i) substantial changes in radiation pattern (e.g., RCPA) and (ii) a large number of radiation pattern states (e.g., RPDA).

We also note that both antenna designs allow for full radiation coverage in the azimuth plane. Therefore, good signal reception is guaranteed independently from the relative orientation of the transmitter and the receiver.

Finally, we remark that the RPDA is characterized by higher radiation efficiency than the RCPA. Thus, we expect the RPDA to collect a stronger signal than the RCPA. This could lead to higher values of channel capacity because of a stronger received SNR, but it could also lead to stronger co-channel interference.

\section{System model and notation}

We assume that the ad hoc network consists of $\mathcal{L}$ colocated links which interfere with each other. All links are single hop (i.e., no node is used for relaying) and all transmit-receive pairs are pre-determined. In the remainder of this article, we will be using the following notations: $\mathbf{H}_{i_{r c} j_{t c}}$ denotes the channel between the receiver of link $i$ and the transmitter of link $j$, which is a function of the receive configuration of link $i\left(i_{r c}\right)$ and the transmit configuration of link $j\left(j_{t c}\right)$; in the case of the RPDA, $l_{r c}, l_{t c} \in[1,4]$ and for the RCPA, $l_{r c}, l_{t c} \in[1$, $2] ; \mathbf{x}_{i}$ is the signal vector of link $i$; operation $(.)^{H}$ denotes the conjugate transpose. Using this notation and assuming a flat fading channel, we can write the input-output relationship for link $l$ as

$$
\mathbf{y}_{l}=\mathbf{H}_{l_{r c}, l_{l c}} \mathbf{x}_{l}+\sum_{i \in \mathcal{L} \backslash l} \mathbf{H}_{l_{r c}, i_{t c}} \mathbf{x}_{i}+\mathbf{n}
$$

where $\sum_{i \in \mathcal{L} \backslash l} \mathbf{H}_{l_{r c}, i_{t c}} \mathbf{x}_{i}+\mathbf{n}$ is the interference plus noise, which results in an interference plus noise covariance matrix for link $l: \mathbf{R}_{l}=\sigma^{2} \mathbf{I}+\sum_{i \in \mathcal{L} \backslash l} \mathbf{H}_{l_{r c}, i_{t c}} \mathbf{Q}_{i} \mathbf{H}_{l_{c c}, i_{c c}}^{H}$. For the above equation, the assumption was made that the noise has power $\sigma^{2}$ and is independent across receive elements. Vector is an $1 \times 2 \mathcal{L}$ vector that contains the 


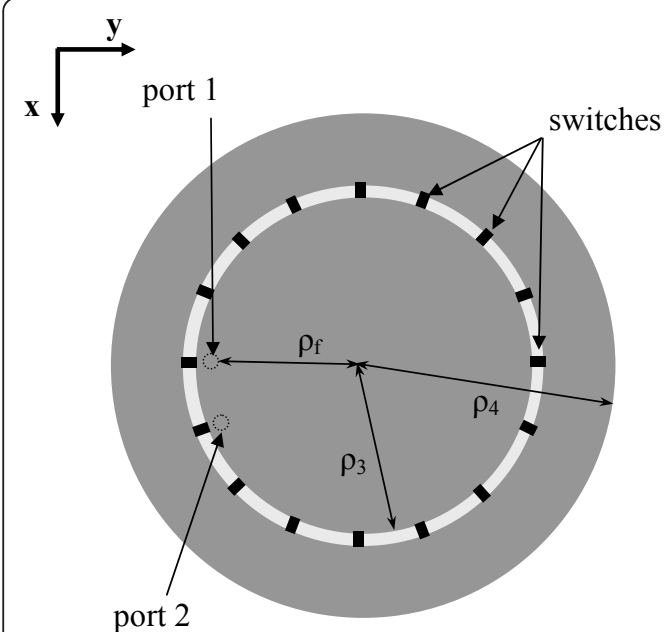

(a)

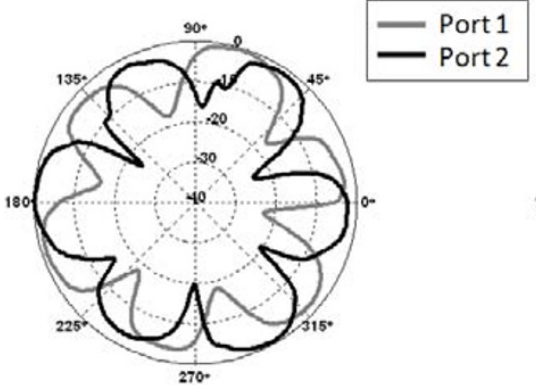

(a)

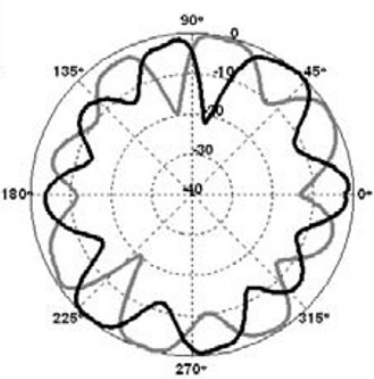

(b)

(b)

Figure 2 (a) Schematic of the reconfigurable circular patch antenna and (b) pattern (in $\mathrm{dB}$ ) in the azimuthal plane at the two ports of the RCPA in all its configurations for an operation frequency of $2.48 \mathbf{~ G H z}$ : (a) port 1,"Mode 3," port 2 "Mode 3"; (b) port 1,"Mode 4," port 2 "Mode 4".

configurations for all links, (i.e., $\mathbf{c}=\left[1_{r c}, 1_{t c}, 2_{r c}, 2_{t c}, \ldots\right.$, $\left.\left.L_{r c}, L_{t c}\right]\right)$. Notice also that the interference plus noise covariance matrix is a function of the receive configuration of the link and the transmit configurations used in the network. We also assume that a single type of reconfigurable antenna, RCPA or RPDA, is used by all nodes in the network.

The power-allocation strategy that we considered in this article is the Equal Power Allocation technique [25]. For the Equal Power Allocation technique, the capacity of link $l$ becomes

$$
C_{l}=\log _{2}\left(\operatorname{det}\left(\mathbf{I}+\frac{P_{T}}{\sigma^{2} N_{T}} \mathbf{H}_{l_{c}, l_{l c}} \mathbf{H}_{l_{r c}, l_{l c}}^{H} \mathbf{R}_{l}^{-1}\right)\right)
$$

where $\mathbf{R}_{l}=\mathbf{I}+\sum_{i \in \mathcal{L} \backslash l} \frac{P_{T}}{\sigma^{2} N_{T}} \mathbf{H}_{l_{r c}, i_{l c}} \mathbf{H}_{l_{r}, i_{t c}}^{H}$ is the interference plus noise covariance matrix.

To quantify the performance of the different types of reconfigurable antennas in an ad hoc network, we use the sum capacity of the network, which is a standard metric for measuring the performance of several colocated interfering links, like in [32]:

$$
C=\sum_{l \in \mathcal{L}} \log _{2}\left(\operatorname{det}\left(\mathbf{I}+\frac{P_{T}}{\sigma^{2} N_{T}} \mathbf{H}_{l_{c}, l_{t c}} \mathbf{H}_{l_{r}, l_{l c}}^{H} \mathbf{R}_{l}^{-1}\right)\right)
$$

Table 4 Spatial correlation between patterns generated at two different ports of the RCPA

\begin{tabular}{cc}
\hline Mode $\mathbf{3}$ & Mode 4 \\
\hline 0.06 & 0.18 \\
\hline
\end{tabular}

Closed-loop MIMO power-allocation algorithms that make use of channel feedback information from the receiver to the transmitter could also be implemented to improve link and network capacities. However, these algorithms become more complex when reconfigurable antennas are used. In particular, channel feedback information would have to be provided for all the different antenna configurations used by the transmitter and receiver. Closed-loop algorithms become even more challenging in a network using reconfigurable antennas because knowledge of the interference state of the network would be needed. This interference also depends on the specific antenna configurations used by all the transmitters in the network, and so it would be difficult to keep all the channel and interference estimates current. Practical channel training aspects were considered by Cetiner in [20]. The closed-loop application of reconfigurable antennas in wireless networks is a topic of future research.

\section{Antenna configuration selection methods}

We consider three different cases for using reconfigurable antennas in the network. In the first case, which we call double-side reconfigurable antennas (DSRA), both the receiver and the transmitter of any given link can

Table 5 Spatial correlation between patterns generated at the same port of the RCPA

\begin{tabular}{ccc}
\hline & $\mathbf{E}_{1, \text { mode 3 }}$ & $\mathbf{E}_{1 \text {,mode 4 }}$ \\
\hline$E_{1, \text { mode 3 }}$ & 1 & 0.2 \\
\hline$E_{1, \text { mode 4 }}$ & 0.2 & 1 \\
\hline
\end{tabular}


Table 6 Measured radiation efficiency of the RCPA

\begin{tabular}{ccc}
\hline & Port 1 (\%) & Port 2 (\%) \\
\hline Mode 3 & 21 & 17 \\
\hline Mode 4 & 6 & 5 \\
\hline
\end{tabular}

adapt its configuration. For the other two cases, either the link receiver or the link transmitter alone is allowed to switch its configuration. We refer to these situations as receiver-side reconfigurable array (RXRA) and transmitter-side reconfigurable array (TXRA), respectively. The side of the link that is not allowed to change configuration is restricted to use the most efficient configuration at all times (i.e., the s-s configuration for the RPDA case and Mode 3 for RCPA). For these three different cases, we consider centralized and a distributed configuration selection schemes, discussed below.

\section{A. Centralized configuration selection technique}

To provide an upper bound on the performance of reconfigurable antennas in ad hoc networks, we consider the use of a powerful centralized controller that has instantaneous knowledge of all communication and interference channels (e.g., $\mathbf{H}_{l_{r c}, i_{c c}}, \forall l, i \in \mathcal{L}$ ). This controller is allowed to control the state of all reconfigurable antennas in the network to optimize the sum capacity given in Equation 4. Specifically, the central controller solves the following optimization problem:

$$
\max _{\mathbf{c}}\left(\sum_{l \in \mathcal{L}} \log _{2}\left(\operatorname{det}\left(\mathbf{I}+\frac{P_{T}}{\sigma^{2} N_{T}} \mathbf{H}_{l_{r c}, l_{t c}} \mathbf{H}_{l_{r c}, l_{t c}} \mathbf{R}_{l}^{-1}\right)\right)\right)
$$

where $\mathbf{c}$ is an $1 \times 2 \mathcal{L}$ ia a vector that contains the configurations for each node. To solve this optimization problem, the centralized controller conducts an exhaustive search over all possible antenna configurations in all the network nodes.

\section{B. Distributed configuration selection technique}

For a more practical approach to configuration selection in MIMO ad hoc networks making use of reconfigurable antennas, we also consider a distributed configuration technique. In this technique, each link makes its own configuration selection using only the link channel $\left(\mathbf{H}_{l_{c}, l_{c}}\right)$ and interference plus noise covariance matrix $\mathbf{R}_{l}$. The assumption of such locally available channel information is commonly used in ad hoc networks (e.g., [7,32-35]). Since each link does not have information about other channels in the network, the antenna configuration decision cannot be geared toward maximizing network sum capacity. Instead, each transmitter performs configuration selection to optimize individual link capacity. Mathematically, link $l$ solves the following optimization problem:

$$
\max _{r c, t c}\left(\log _{2}\left(\operatorname{det}\left(\mathbf{I}+\frac{P_{T}}{\sigma^{2} N_{T}} \mathbf{H}_{l_{r c}, l_{t c}} \mathbf{H}_{l_{r c}, l_{t c}} \mathbf{R}_{l}^{-1}\right)\right)\right)
$$

where $\mathbf{R}_{l}$ continues to depend on the transmit configuration of all the other links and the receive configuration of link $l$. However, a change in transmit configuration for a particular link leads to a different amount of interference encountered by the other links. These other links, in turn, will have to respond to this change in interference levels by choosing their antenna configurations to maximize their own capacity. Thus, the Distributed technique is an iterative procedure where each link continually updates its configuration selection in response to changes in the interference. The procedure is very similar to the Iterative Waterfilling [32,35] algorithm, but instead of using different power allocation matrices to respond to changes in the interference, the nodes will use different antenna configuration combinations.

\section{Single side reconfigurable antennas}

As mentioned previously, we individually considered situations with reconfigurable antennas at both ends of the link (DSRA), at the transmitter only (TXRA) and at the receiver only (RXRA). Looking at Equation 3, we note that a link's capacity is a function of receive and transmit configurations of it, as well as the transmit configurations of the other links (through the interference plus noise covariance matrix R) that coexist in the network. In other words, when a link changes its receive configuration, it affects only its own capacity, while when a link changes its transmit configuration, it does not only affect its own capacity but also the capacities of all the other links. Therefore, in the case of distributed antenna configuration selection, when a link is allowed to change its transmit configuration (i.e., TXRA and DSRA), there is the need for an iterative procedure, so as to allow for the other links to respond to the new interference levels caused by transmit configuration changes. However, when only the receive configurations are allowed to change (i.e., RXRA), a change of the configuration in one link will affect only this link, and thus iterations are no longer needed.

Apart from the inherent iterative nature of the TXRA and DSRA configuration selection schemes, allowing configuration changes in the receive side only has another positive outcome: it removes the requirement of having to implement a feedback loop from the receiver to the transmitter, which communicates the configuration which it should be using. These two properties make the RXRA scheme much more appealing for a practical implementation because of its simplicity and lower overhead. 
The RXRA technique is also desirable in that the Distributed and Centralized schemes become equivalent; when a link maximizes its own capacity by changing reconfigurable antennas only at the receiver, it also maximizes network sum capacity. Distributed and Centralized schemes are not necessarily equivalent when reconfigurable antennas are used at the transmitters of ad hoc network links as the "selfish" choice that each node makes in a distributed scheme to maximize its own capacity, is no longer guaranteed to have a positive impact on the overall network sum capacity.

Configuration adaptation at a single side of the link also provides a smaller search space for the Centralized technique and less channel training for the Distributed technique. For example, in the case of RPDAs where four configurations are available, a link has 16 different configuration combinations to choose from with DSRA. However, this number decreases to four configuration combinations for TXRA and RXRA. This difference in the number of available configurations, while reducing the degrees of freedom the network has, would also require less training. Less channel training may have a positive impact on the performance when the channel estimation errors are taken into account[21], depending on the total number of configuration combinations that need to be considered.

When assuming configuration adaptation at only one side of the link, we still assume that the other link end uses a reconfigurable antenna, since in an ad hoc network, any node can be either a receiver or a transmitter. However, the side that is not allowed to switch its configuration is restricted to use the most radiation-efficient configuration at all times.

\section{Data Collection}

The performance that can be achieved, in terms of sum network capacity, combining reconfigurable antennas and the techniques described in Section 4, was investigated through field measurements and electromagnetic ray-tracing simulations in an indoor environment.

\section{A. Measurement setup}

Our measurement campaign took place in the third floor of the Bossone building on Drexel campus, wherein the Drexel Wireless Systems Laboratory (DWSL) is located. For the measurements, we used the HYDRA Software Defined Radio platform [36]. This platform was also used for the evaluation of reconfigurable antennas in single link scenarios [18,28]. HYDRA is a $2 \times 2$ MIMO platform that operates in the $2.4-\mathrm{GHz}$ band using OFDM with 64 subcarriers (52 are carrying data).

Two RCPAs and four RPDAs were built and equipped with pin diodes for the measurements, so as to be used in both the receiver and the transmitter. The radiation patterns of these antennas were measured in an anechoic chamber so as to be used for the simulation procedure, explained in Section 5-B.

The network measurement topology is shown in Figure 3. Three nodes (RX1 to RX3) with two receive elements each acted as receivers, and three nodes (TX1 to TX3) with two transmit elements each acted as transmitters, so as to create six different network topologies, by perturbating the intended receiver-transmitter pairs. To capture small-scale fading effects, the receive elements were placed on a robotic antenna positioner and were moved at 40 different positions at displacements of $\lambda / 10$ along the $y$-axis for RX1 and RX2, and along the $x$-axis for RX3. At each position, 100 noisy channel estimates were captured and averaged for each subcarrier, so as to get the channel response between each receiver-transmitter pair. Each receiver-transmitter pair channel's response was measured separately as described, and superposition of fields was employed to recreate an interference-limited network. Based on these estimated channels for each of the positions, the sum network capacity was calculated as discussed in Section 4. In this way, we acquired 240 samples (6 network topologies with 40 samples each) of sum network capacities per subcarrier for each of the employed antennas and each configuration selection scheme. The response at each subcarrier was treated as an independent narrow band channel and, for each location, the sum network capacity was averaged over these 52 subcarriers.

The acquired channels were normalized with a common parameter, so that $\max _{l, i \in \mathcal{L} r c, t c} E\left\{\left.\sum_{s=1}^{52}\left|\mathbf{H}_{l_{c}, i_{t c}}^{s}\right|\right|_{F} ^{2}\right\}=4 \cdot 52$, with the expectation over the 40 positions and subcarrier index, $s$. This normalization was performed on a per reconfigurable antenna basis (i.e., one normalization parameter for the RCPA, and one for the RPDA) because of the large difference in radiation efficiency between the two antenna architectures.

\section{B. Simulation setup}

The simulated channels were acquired via numerical computation using an electromagnetic ray tracer, FASANT [37]. A 3D model of the hallway of the third floor of the Bossone Research building on Drexel University campus was simulated as the geometry input of FASANT.

The measured 3D radiation patterns of the two antennas presented in Section 2 were used in the ray-tracing simulation both at the receiver and at the transmitter in a $2 \times 2$ MIMO ad hoc network. Note that the orientation of the reconfigurable antennas was selected such that the maximum degree of pattern diversity between 


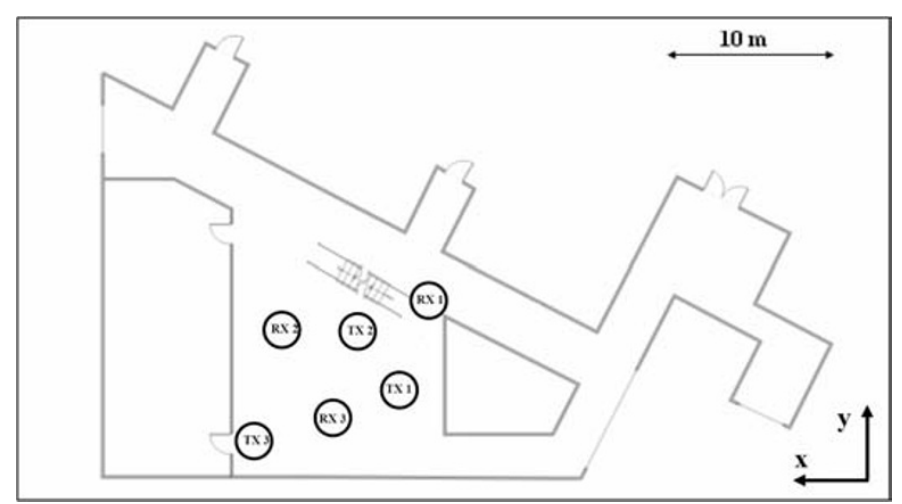

Figure 3 Measured topology.

the patterns of different antenna configurations was in the azimuthal plane.

The simulations were conducted by transmitting a single tone at $2.484 \mathrm{GHz}$ to obtain the values of the entries of the channel matrices, $\mathbf{H}$, for all channel and interference matrices. The extracted channel matrices were then utilized to calculate the sum network capacity for each of the methods discussed in Section 4.

The simulated channels, as in the measurement case, were normalized with a common parameter, so that $\max _{l, i \in \mathcal{L}, r c, t c} E\left\{\mid \mathbf{H}_{l_{r c}, i_{t c}}^{s} \|_{F}^{2}\right\}=4$, with the expectation over the 40 positions. Again, like measurements, one normalization factor was used for the RPDA, and another normalization factor was used for the RCPA.

We would like to point out that the 3D model used for the simulations was not able to capture all the details of the actual measurement environment; namely, only two different materials were used: one for the floor, and one of the walls, and other details (e.g., small furniture in the environment, structure behind the drywall, and an elevator shaft behind the wall on the right of transmitter 1) were not incorporated. Although these modeling deficiencies did not allow for an exact match between measurements and simulations, there was still very good agreement in most of the cases as will be shown in Section 6.

\section{Results}

For the following results, we assumed that $\frac{P_{T}}{\sigma^{2}}=100$ for all the nodes. The maximum number of iterations allowed for the Distributed TXRA and DSRA techniques was 10. If convergence was still not achieved after 10 iterations, the sum capacity achieved at the 10th iteration was used in forming the CDFs that appear in Sections 6-A1 and 6-B1. However, when the Distributed TXRA and DSRA techniques did not converge, the iteration count was not included in the calculation of the average number of iterations discussed in Sections
6-A2 and 6-B2. The maximum number of iterations allowed was determined arbitrarily, based on the fact that the vast majority of instances did converge in less than three iterations, as explained in Sections 6-A2 and 6-B2.

\section{A. RCPA}

\section{1) Sum capacity results}

In Figures $4 \mathrm{a}$ and $4 \mathrm{~b}$, the CDFs of the network sum capacity using the Centralized configuration selection methods are plotted for the measured and simulated results, respectively. The CDFs of sum capacity resulting from the Distributed configuration selection schemes appear in Figure 4c and 4d for the measurement results and the simulation results, respectively. Both the simulation and measurement CDFs show that the increases in sum capacity, as compared with the case where all nodes are equipped with non-reconfigurable Mode 3 circular patch antennas, are considerable. For easier comparison, the expected sum capacity resulting from these CDFs and the capacity percentage increase of using reconfigurable antennas are summarized in Table 7 . From this table, we can observe that the measured sum capacity increases are greater than those predicted from the simulations. In particular, for the Centralized DSRA scheme, simulations show an increase of around 50\% when using reconfigurable antennas, whereas for the measurements, the percentage increase is around $75 \%$. Note that both simulations and measurements show that relatively large sum capacity increases can be expected-the minimum increase is $8.70 \%$ for the measured Distributed TXRA case, while for the more appealing Distributed RXRA technique (Section 4-C), the percentage increase is $14 \%$ for the simulations and $31 \%$ for the measurements. The trends in selection technique performance are generally the same for both the measured and simulated results. However, in the Distributed RXRA and TXRA techniques, the trends are 


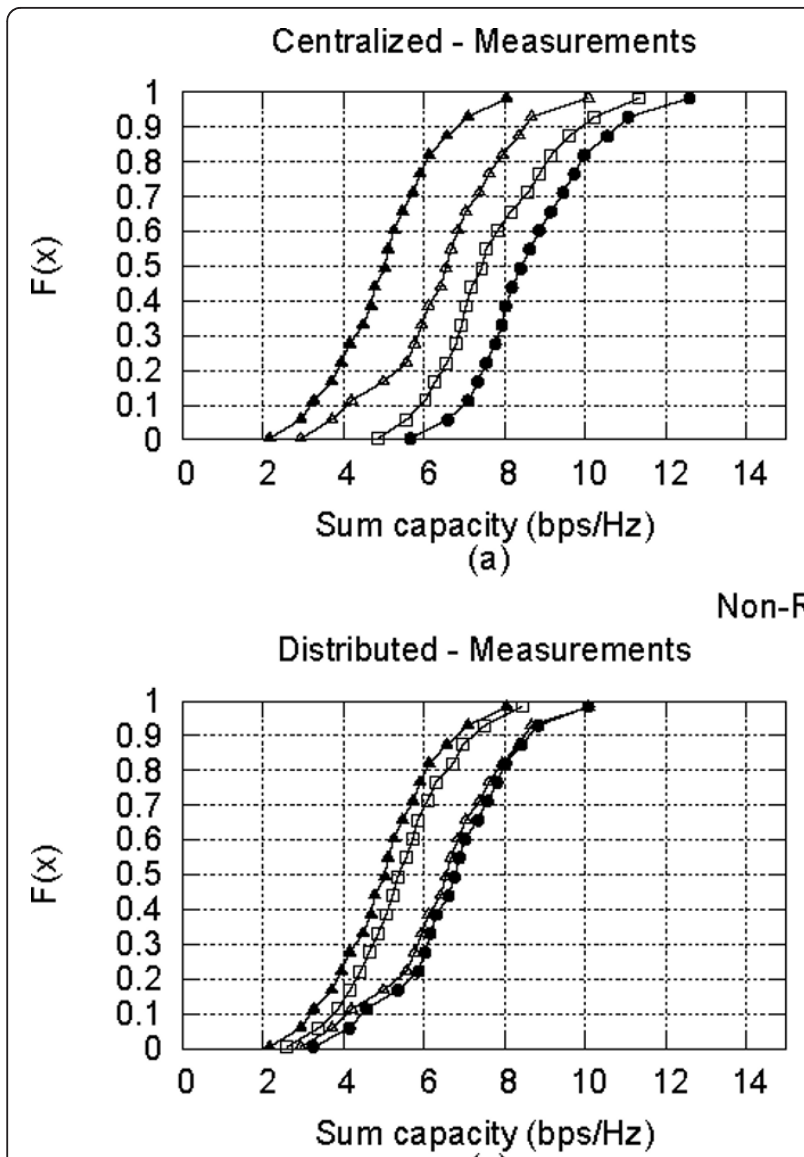

(c)

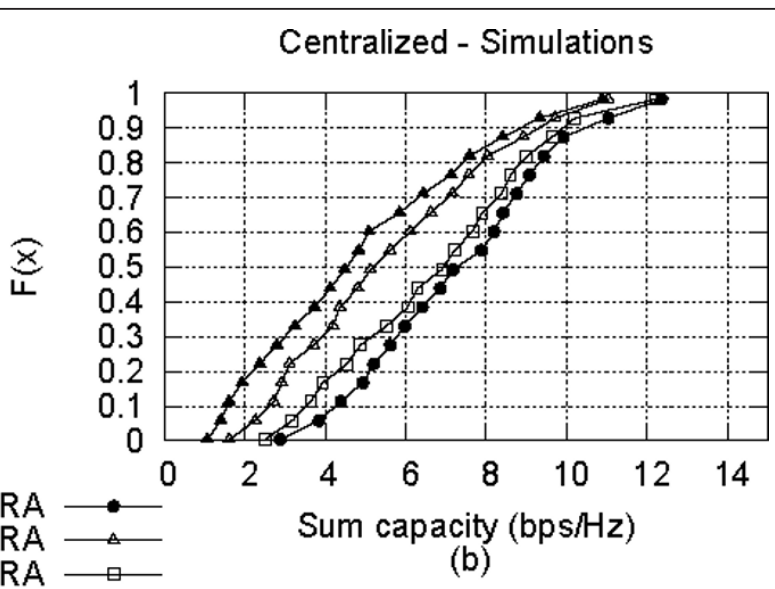

(b)

Figure 4 CDF of sum capacity for RCPA with equal power allocation. (a) Measurements of centralized selection, (b) simulations of centralized selection, (c) measurements of distributed selection and (d) simulations of distributed selection.

Table 7 RCPA mean sum network capacity

\begin{tabular}{lcc}
\hline Selection technique & Mean sum capacity $(\mathbf{b p s} / \mathbf{H z})$ & \% Increase vs. non-reconfigurable \\
\hline DSRA-distributed & 6.40 & 30.77 \\
\hline RXRA-distributed & 5.60 & 14.42 \\
\hline TXRA-distributed & 5.91 & 20.93 \\
\hline DSRA-centralized & 7.33 & 49.90 \\
\hline RXRA-centralized & 5.60 & 14.42 \\
\hline TXRA-centralized & 6.79 & 38.80 \\
\hline Non-reconfigurable & 4.89 & 0 \\
\hline & Measurements & 35.51 \\
\hline DSRA-distributed & 6.84 & 30.81 \\
\hline RXRA-distributed & 6.60 & 8.70 \\
\hline TXRA-distributed & 5.49 & 75.68 \\
\hline DSRA-centralized & 8.87 & 30.81 \\
\hline RXRA-centralized & 6.60 & 55.11 \\
\hline TXRA-centralized & 7.83 & 0 \\
\hline Non-reconfigurable & 5.05 & \\
\hline
\end{tabular}


reversed: in the measurements Distributed RXRA outperforms Distributed TXRA, whereas, in simulations, the reverse is true.

\section{2) Convergence properties}

Table 8 shows the average number of iterations required before convergence for the iterative Distributed DSRA and Distributed TXRA techniques. From this table, we can observe that convergence is achieved quickly, even in the DSRA case, where both the transmitter and receiver were adapting their antenna configurations. Recall that scenarios in which there was no convergence after 10 iterations were not included in the average shown in Table 8. However, in both measurements and simulations, more than $99 \%$ of the scenarios reached convergence before the 10th iteration. To be more specific, in the simulations, $0.42 \%$ scenarios did not converge before 10 iterations for the DSRA case and $0.83 \%$ for TXRA case. For the measurement case, for both DSRA and TXRA, only $0.08 \%$ of the cases did not converge.

\section{B. RPDA}

\section{1) Sum capacity results}

The network sum capacity CDFs for the Centralized selection schemes when the nodes are equipped with RPDAs appear in Figure $5 \mathrm{a}$ and $5 \mathrm{~b}$ for measurements and for simulations, respectively. The corresponding sum capacity CDFs when the configuration selection is performed in a Distributed manner appear in Figure 5c and $5 \mathrm{~d}$ for the measurements and for the simulations, respectively. In Table 9, the expected sum capacities resulting from these CDFs are gathered together with the percentage increase in expected network sum capacity versus the non-reconfigurable case, where all the nodes were equipped with dipoles in the S-S configuration. As in the RCPA results, we again see that the simulations underestimated the performance increase that was observed using the measurement results. However, for the RPDA results, the relative performance between the configuration selection schemes is maintained between measurements and simulations, with the Centralized DSRA technique performing the best, and the Distributed TXRA technique performing the worst of all techniques using reconfigurable antennas. By comparing these results with the RCPA results in the previous section, we can see that in both simulations and

Table 8 Average number of iterations before convergence

\begin{tabular}{llcc}
\hline Antenna & Selection technique & Simulations & Measurements \\
\hline RCPA & DSRA-distributed & 2.1 & 1.9 \\
\hline RCPA & TXRA-distributed & 1.7 & 1.2 \\
\hline RPDA & DSRA-distributed & 2.5 & 3.0 \\
\hline RPDA & RXRA-distributed & 2.0 & 2.3 \\
\hline
\end{tabular}

measurements, RPDAs provide a larger percentage increase in capacity than RCPAs. Furthermore, we can see that the worst we can expect as a percentage increase in sum capacity relative to non-reconfigurable antennas is $10 \%$ for the simulated TXRA technique and $30 \%$ for the measured TXRA technique. For the desirable Distributed RXRA scheme discussed in Section 4$\mathrm{C}$, there is a simulated increase in capacity of $24 \%$ and an increase of $31 \%$ in measured capacity relative to nonreconfigurable antennas.

\section{2) Convergence properties}

The two iterative configuration selection schemes using RPDAs needed on average more iterations before convergence than the RCPA case, as shown in Table 8 . This longer convergence time can be attributed to the fact that RPDAs have more configurations to choose from than the RCPAs. The greater number of configurations to choose from also increased the number of scenarios in which there was no convergence after 10 iterations. In particular, for the measurement data, in the Distributed DSRA case, $26 \%$ of the scenarios did not converge before 10 iterations. Similarly, in the Distributed TXRA case, $7 \%$ of the scenarios did not converge before 10 iterations. For the simulations, all of the scenarios reached convergence ( $0 \%$ did not converge) for both DSRA and TXRA. While it would certainly have been possible to continue the iterative process until convergence was achieved, we chose to limit the number of iterations to 10 before stopping the configuration update process because a practical system would not have an indefinite amount of time for configuration selection before network information became outdated.

\section{Comparing RCPA with RPDA}

A direct comparison of the performance of the RPDA and the RCPA, when employed in an ad hoc network shows that the performance of the RPDA is higherboth in percentage increase relative to non-reconfigurable architectures and in absolute sum network capacity values. The performance of a reconfigurable antenna array should be a function of the following factors: (i) the number of configurations available, (ii) the pattern diversity between different configurations, and (iii) the relative efficiency between the different configurations. While the relative radiation efficiency between RPDA and RCPA is important, the normalization process, described in Section 5, effectively sets the efficiency of RPDA configuration S-S equal to RPCA configuration Mode 3. If we had not performed this normalization, then a direct comparison between the two architectures would not have been possible, since RPDA efficiency is much higher than that of the RCPA.

The superior performance of the RPDA, as compared with the RCPA, can be explained by the fact that the 


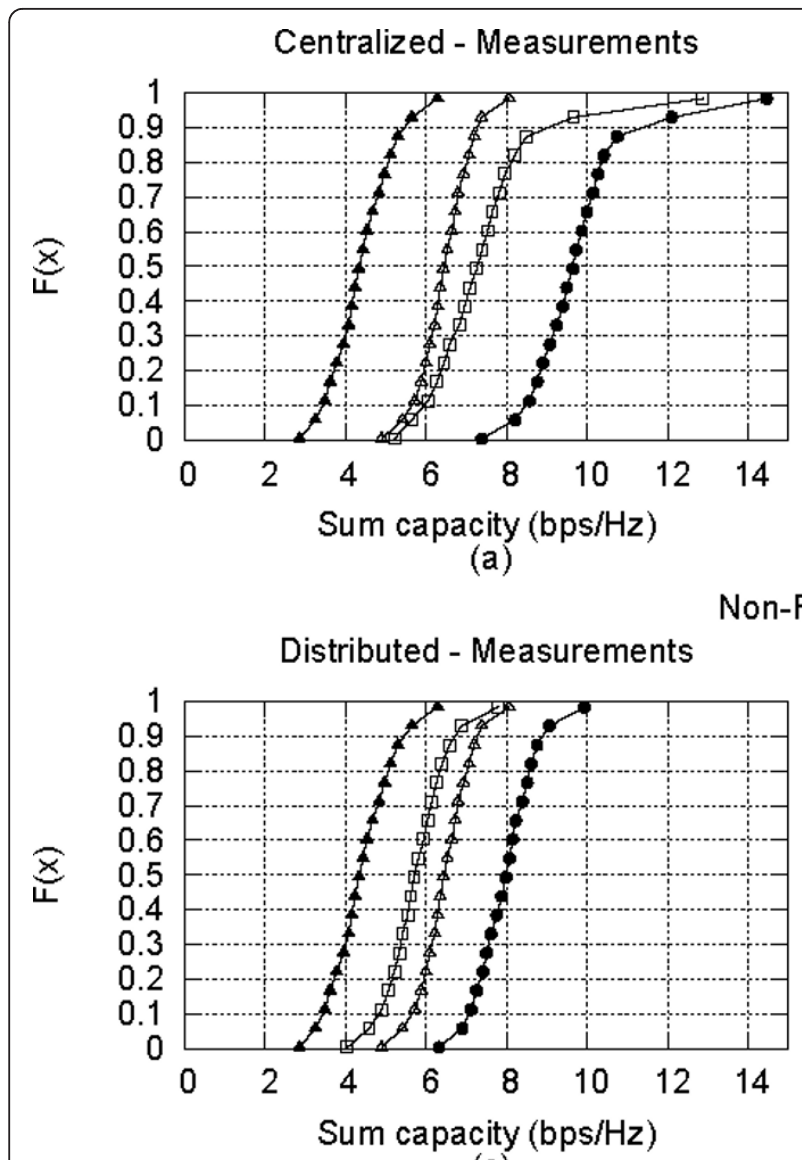

(c)

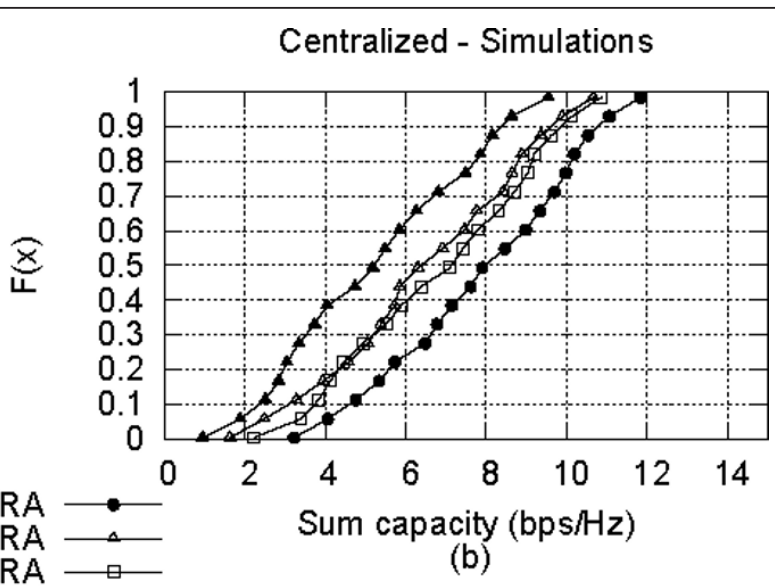

(b)

Figure 5 CDF of sum capacity for RPDA with equal power allocation. (a) Measurements of centralized selection, (b) simulations of centralized selection, (c) measurements of distributed selection and (d) simulations of distributed selection.

Table 9 RPDA mean sum network capacity

\begin{tabular}{llc}
\hline Selection technique & Mean sum capacity $(\mathbf{b p s} / \mathbf{H z})$ & \% Increase vs. non-reconfigurable \\
\hline DSRA-distributed & 6.83 & 30.40 \\
\hline RXRA-distributed & 6.51 & 24.38 \\
\hline TXRA-distributed & 5.78 & 10.31 \\
\hline DSRA-centralized & 7.91 & 51.04 \\
\hline RXRA-centralized & 6.51 & 24.38 \\
\hline TXRA-centralized & 6.85 & 30.79 \\
\hline Non-reconfigurable & 5.23 & 0 \\
\hline & Measurements & 81.42 \\
\hline DSRA-distributed & 8.00 & 46.85 \\
\hline RXRA-distributed & 6.48 & 30.71 \\
\hline TXRA-distributed & 5.77 & 122.76 \\
\hline DSRA-centralized & 9.83 & 46.85 \\
\hline RXRA-centralized & 6.48 & 69.42 \\
\hline RXRA-centralized & 7.48 & 0 \\
\hline Non-reconfigurable & 4.41 & \\
\hline
\end{tabular}


RPDA has more configurations available (4 configurations per array as opposed to 2 for the RCPA) and that its configurations are closer to each other in terms of efficiency (i.e., RPDA efficiency varies from 84 to $48 \%$ as opposed to the RCPA, where the efficiency varies from 21 to $5 \%)$. On the other hand, the RCPA does have an advantage in that the radiation patterns of all the available configurations show very low correlation (Table 5).

\section{Effect of the number of configurations}

In order to better analyze the effects of the number of available configurations, we calculated the sum network capacity for the case where the RPDAs were only allowed to switch between the S-S and the L-L configurations. In this case, we are able to gain insight into the importance of having a large number of array configurations. In this situation, the RPDA has as many configuration settings as the RCPA, but with radiation patterns that are highly correlated (Table 2). Comparing Table 10 with Table 9 from Section 6-B1, we see that the percentage capacity increase relative to the nonreconfigurable case was almost halved for both measurements and simulations when the RPDA was restricted in switching only between the S-S and L-L configurations. These results highlight the importance of having a large number of antenna configurations to switch between, even if these configurations have radiation patterns that are relatively highly correlated.

We can also observe that the RCPA performs better, in absolute numbers, than the RPDA when the RPDA is confined to using only two of the available configurations. This result holds true even though the radiation efficiency difference between S-S configuration and $\mathrm{L}-\mathrm{L}$ configuration is smaller than the radiation efficiency difference between Modes 3 and 4 of the RCPA. This result is due to the smaller correlation that exists between Mode 3 and 4 patterns in the RCPA, as compared to the correlation between S-S and L-L patterns in the RPDA. The effect of uncorrelated patterns will be considered in more detail in the next section.

\section{E. Effect of correlation between the patterns}

In this section, we introduce a new normalization procedure to isolate the effect of correlation between the radiation patterns in reconfigurable antennas. In particular, we normalized each antenna configuration separately, so that the maximum expected squared Frobenious norm between the channels with the same configuration combination would be the same. Thus, there are four normalization factors for the "reduced" RPDA discussed in the previous sub-section (i.e., one for (S-S)-(S-S), another for (S-S)-(L-L), etc). Similarly, there are four normalization factors for the RCPA (i.e., one for Mode 3-Mode 3, another for Mode 3-Mode 4, etc.). In this way, we removed the effects of radiation efficiency, forcing all configuration combinations to "receive" the same power, while keeping the relative channel strengths of the different links in the topology. Mathematically, the normalization parameter for the case where the receiver was using configuration $r x$ and the transmitter was using configuration $t x$ was chosen such that $\max _{l, i} E\left\{\left|\mathbf{H}_{l_{r}, i_{t} c}\right|_{F}^{2}\right\}=4$ for simulations and

Table 10 Mean sum network capacity-RPDA results using SS and LL configurations

\begin{tabular}{lcc}
\hline Selection technique & Mean sum capacity $(\mathbf{b p s} / \mathbf{H z})$ & \% Increase vs. non-reconfigurable \\
\hline DSRA-distributed & 5.93 & 13.3 \\
\hline RXRA-distributed & 5.84 & 11.58 \\
\hline TXRA-distributed & 5.38 & 2.76 \\
\hline DSRA-centralized & 6.60 & 26.06 \\
\hline RXRA-centralized & 5.84 & 11.58 \\
\hline TXRA-centralized & 6.04 & 15.31 \\
\hline Non-reconfigurable & 5.23 & 0 \\
\hline & \multicolumn{1}{c}{ Measurements } & 45.23 \\
\hline DSRA-distributed & 6.41 & 24.70 \\
\hline RXRA-distributed & 5.50 & 20.73 \\
\hline TXRA-distributed & 5.33 & 65.37 \\
\hline DSRA-centralized & 7.3 & 24.70 \\
\hline RXRA-centralized & 5.50 & 41.06 \\
\hline RXRA-centralized & 6.23 & 0 \\
\hline Non-reconfigurable & 4.41 & \\
\hline
\end{tabular}


Table 11 Mean sum capacity for patterns normalized separately with RPDA using only S-S and L-L configurations

\begin{tabular}{lll}
\hline Selection technique & RPDA mean sum capacity (bps/Hz) & RCPA mean sum capacity (bps/Hz) \\
\hline DSRA distributed & 6.43 & 7.13 \\
\hline RXRA distributed & 6.01 & 6.07 \\
\hline TXRA distributed & 5.54 & 5.91 \\
\hline DSRA centralized & 6.85 & 8.05 \\
\hline RXRA centralized & 6.01 & 6.07 \\
\hline TXRA centralized & 5.97 & 6.84 \\
\hline Non-reconfigurable & 5.23 & 4.89 \\
\hline & Measurements & \\
\hline DSRA distributed & 6.42 & 8.12 \\
\hline RXRA distributed & 5.51 & 6.80 \\
\hline TXRA distributed & 5.35 & 6.12 \\
\hline DSRA centralized & 7.31 & 9.17 \\
\hline RXRA centralized & 5.51 & 6.80 \\
\hline TXRA centralized & 6.23 & 7.52 \\
\hline Non-reconfigurable & 4.41 & 5.05 \\
\hline
\end{tabular}

$\max _{l, i} E\left\{\sum_{s=1}^{52}\left|\mathbf{H}_{l_{r} c, i_{t} c}\right|_{F}^{2}\right\}=4 \cdot 52$ for measurements, with the expectation taken along the 40 points.

The RPDA performance was again considered for the case where only the S-S and the L-L configurations were used. In this way, we can compare the performance of two reconfigurable antenna array structures, with each having two configurations available and with all the configurations having the same radiation efficiency. The only difference between the two structures is the correlation between the available configurations. The RCPA structure exhibits almost uncorrelated patterns (Table 5), while the RPDA configurations are highly correlated (Table 2). The calculated expected sum network capacities are shown in Table 11.

From this table, we can observe that the less-correlated patterns that the RCPA offers significantly improves the expected sum capacity. We can also observe that the capacity values for the RPDA do not change much with this new normalization, unlike the RCPA values, whose mean sum capacity values are significantly improved by forcing both modes to receive the same power. These results show that uncorrelated radiation patterns, as well as the number of configurations and relative radiation efficiency, can be a mechanism through which reconfigurable antennas enhance ad hoc networks.

\section{Conclusions}

In this study, we have studied the performance of two different reconfigurable antenna structures when employed in a MIMO ad hoc network. We have investigated the cases where reconfigurable antennas are employed at both link ends, as well as at either the receiver or transmitter. We quantified the performance of these cases with both a Centralized and Distributed configuration selection scheme. For all of the investigated techniques, we have quantified the great capacity increases that can be expected using reconfigurable antennas in a MIMO ad hoc network. We have also provided insight into the design of reconfigurable antenna arrays, by quantifying the effects of the number of configurations available, the correlation between different configurations, as well as the effect of radiation efficiency differences between the different configurations. Our results show that a reconfigurable antenna designer should aim at designing an antenna that can provide a relatively large number of available configurations with as high pattern diversity as possible, but should also aim at keeping the radiation efficiency of the different configurations balanced. The Distributed technique in which only the receiver is allowed to switch configurations (i.e., RXRA) was shown to strike a good balance between sum network capacity increases and practical channel feedback and network information constraints.

\section{Acknowledgements \\ A previous version of this article appeared in the Proceedings of the 2008 Radio and Wireless Symposium.}

\section{Author details}

'Department of Electrical and Computer Engineering, Drexel University, USA

${ }^{2}$ Dipartimento di Elettronica e Informazione Politecnico Di Milano, Italy 


\section{Competing interests}

The authors Daniele Piazza, Michele D'Amico and Kapil R Dandekar are Cofounders of Adant, a company whose mission is to develop advanced, innovative antennas for wireless communication systems using intellectual property related to this publication. John Kountouriotis has worked as a contractor for Adant.

Received: 2 December 2010 Accepted: 31 October 2011

Published: 31 October 2011

\section{References}

1. S Bellofiore, J Foutz, R Govindarajula, I Bahceci, C Balanis, A Spanias, J Capone, T Duman, Smart antenna system analysis, integration and performance for mobile ad-hoc networks (MANETs). IEEE Transactions on Antennas and Propagation. 50(5), 571-581 (2002). doi:10.1109/ TAP.2002.1011222

2. R Ramanathan, J Redi, C Santivanez, D Wiggins, S Polit, Ad hoc networking with directional antennas: a complete system solution. IEEE Journal on Selected Areas in Communications. 23(3), 496-506 (2005)

3. T Ohira, Emerging adaptive antenna techniques for wireless ad-hoc networks, in ISCAS 2001 The 2001 IEEE International Symposium on Circuits and Systems. 4, 858-861 (2001)

4. H Ming, Z Junshan, MIMO ad hoc networks with spatial diversity: medium access control and saturation throughput, in 2004 43rd IEEE Conference on Decision and Control. 3, 3301-3306 (2004)

5. M Park, R Heath Jr, S Nettles, Improving throughput and fairness for MIMO ad hoc networks using antenna selection diversity, in GLOBECOM 'O4 IEEE Global Telecommunications Conference. 5, 3363-3367 (2004)

6. A Nasipuri, S Ye, J You, R Hiromoto, A MAC protocol for mobile ad hoc networks using directional antennas, in 2000 IEEE Wireless Communications and Networking Conference. 3, 1214-1219 (2000)

7. R Iltis, S Kim, D Hoang, Noncooperative iterative MMSE beamforming algorithms for ad hoc networks, in IEEE Transactions on Communications. 54(4), 748-759 (2006)

8. S Bellofiore, J Foutz, C Balanis, A Spanias, Smart-antenna system for mobile communication networks part 2: Beamforming and network throughput, in IEEE Antennas and Propagation Magazine. 44(4), 106-114 (2002). doi:10.1109/MAP.2002.1043158

9. R Ramanathan, On the performance of ad hoc networks with beamforming antennas, in Proceedings of the 2001 ACM International Symposium on Mobile Ad Hoc Networking and Computing, 95-105 (2001)

10. R Mailloux, Phased array antenna handbook (Artech House, 1994)

11. S Bellofiore, C Balanis, J Foutz, A Spanias, Smart-antenna systems for mobile communication networks. part 1: Overview and antenna design, in IEEE Antennas and Propagation Magazine. 44(3), 145-154 (2002). doi:10.1109/ MAP.2002.1039395

12. J Cheng, M Hashiguchi, K ligusa, T Ohira, Electronically steerable parasitic array radiator antenna for omni- and sector pattern forming applications to wireless ad hoc networks. IEE Proceedings-Microwaves, Antennas and Propagation. 150(4), 203-208 (2003). doi:10.1049/ip-map:20030548

13. R Vaughan, Switched parasitic elements for antenna diversity. IEEE Transactions on Antennas and Propagation. 47(2), 399-405 (1999). doi:10.1109/8.761082

14. C Jaikaeo, CC Shen, Multicast communication in ad hoc networks with directional antennas, in Proceedings 12th International Conference on Computer Communications and Networks, 385-390 (2003)

15. SD Blostein, H Leib, Multiple antenna systems: Their role and impact in future wireless access. IEEE Communications Magazine. 41(7), 94-101 (2003). doi:10.1109/MCOM.2003.1215645

16. D Piazza, N Kirsch, A Forenza, R Heath Jr, K Dandekar, Design and evaluation of a reconfigurable antenna array for MIMO systems. IEEE Transactions on Antennas and Propagation. 56(3), 869-881 (2008)

17. JD Boerman, JT Bernhard, Performance study of pattern reconfigurable antennas in MIMO communication systems. IEEE Transactions on Antennas and Propagation. 56(1), 231-236 (2008)

18. D Piazza, P Mookiah, M D'Amico, K Dandekar, Two port reconfigurable circular patch for MIMO systems, in Proceedings of the 2007 European Conference on Antennas and Propagation (EUCAP) (2007)

19. BA Cetiner, H Jafarkhani, J-Y Qian, HJ Yoo, A Grau, F De Flaviis, Multifunctional reconfigurable MEMS integrated antennas for adaptive MIMO systems, in IEEE Communications Magazine. 42(12), 62-70 (2004)
20. B Cetiner, E Akay, E Sengul, E Ayanoglu, A MIMO system with multifunctional reconfigurable antennas. IEEE Antennas and Wireless Propagation Letters. 5(31), 463-466 (2006)

21. A Grau, H Jafarkhani, F De Flaviis, A reconfigurable multiple-input multipleoutput communication system. Wireless Communications, IEEE Transactions on. 7, 1719-1733 (May 2008)

22. A Sayeed, V Raghavan, Maximizing MIMO capacity in sparse multipath with reconfigurable antenna arrays. IEEE Journal of Selected Topics in Signal Processing. 1 (June 2007)

23. H Eslami, C Sukumar, D Rodrigo, S Mopidevi, A Eltawil, L Jofre, B Cetiner, Reduced overhead training for multi reconfigurable antennas with beamtilting capability, in IEEE Transactions on Wireless Communications. 9(12), 3810-3821 (2010)

24. D Piazza, J Kountouriotis, M D'Amico, K Dandekar, A technique for antenna configuration selection for reconfigurable circular patch arrays, in IEEE Transactions on Wireless Communications. 8(3), 1456-67 (2009)

25. E Telatar, Capacity of multi-antenna gaussian channels. European Transactions on Telecommunications. 10(6), 585-595 (1999). doi:10.1002/ ett.4460100604

26. C Waldschmidt, J Hagen, W Wiesbeck, Influence and modelling of mutual coupling in MIMO and diversity systems, in IEEE Antennas and Propagation Society, AP-S International Symposium (Digest). 3, 190-193 (2002)

27. R Andersen, J Vaughan, Antenna diversity in mobile communications. IEEE Transactions on Vehicular Technology. T-36(4), 149-172 (1987)

28. D Piazza, K Dandekar, Reconfigurable antenna solution for MIMO-OFDM systems. Electronics Letters. 42(8), 15-16 (2006)

29. CA Balanis, Antenna Theory: Analysis and Design (Wiley, 1997)

30. R Vaughan, Two-port higher mode circular microstrip antennas. IEEE Transactions on Antennas and Propagation. 36(3), 309-21 (1988). doi:10.1109/8.192112

31. I Bahl, P Bhartia, Microstrip Antennas, (Artech House, 1980)

32. S Ye, RS Blum, Optimized signaling for MIMO interference systems with feedback. IEEE Transactions on Signal Processing. 51(11), 2839-2848 (2003). doi:10.1109/TSP.2003.818339

33. F Farrokhi, G Foschini, A Lozano, R Valenzuela, Link-optimal BLAST processing with multiple-access interference, in IEEE Vehicular Technology Conference. 1(52D), 87-91 (2000)

34. RS Blum, MIMO capacity with interference. IEEE Journal on Selected Areas in Communications. 21(5), 793-801 (2003). doi:10.1109/JSAC.2003.810345

35. M Demirkol, M Ingram, Power-controlled capacity for interfering MIMO links, in IEEE Vehicular Technology Conference. 1(54D), 187-191 (2001)

36. A Gupta, A Forenza, RW Heath Jr, Rapid MIMO-OFDM software defined radio system prototyping. IEEE Workshop on Signal Processing Systems, SiPS: Design and Implementation, Austin, TX, United States 182-187 (2004)

37. MF Catedra, J Perez, A Gonzalez, O Gutierrez, F Saez de Adana, Fast computer tool for the analysis of propagation in urban cells, in Annual Wireless Communications Conference, Proceedings, 240-245 (1997)

\section{doi:10.1186/1687-1499-2011-147}

Cite this article as: Kountouriotis et al: Reconfigurable antennas and configuration selection methods for MIMO ad hoc networks. EURASIP Journal on Wireless Communications and Networking 2011 2011:147.

\section{Submit your manuscript to a SpringerOpen ${ }^{\mathcal{O}}$ journal and benefit from:}

- Convenient online submission

- Rigorous peer review

- Immediate publication on acceptance

- Open access: articles freely available online

- High visibility within the field

- Retaining the copyright to your article

Submit your next manuscript at $\boldsymbol{~ s p r i n g e r o p e n . c o m ~}$ 